D.O.I.: $10.3895 / \mathrm{S} 1808-04482009000400004$

\title{
INFLUÊNCIAS DA ESTRATÉGIA DE PRODUÇÃO NA GESTÃO DA CADEIA DE SUPRIMENTOS: UMA ABORDAGEM BASEADA EM PROCESSOS DE NEGÓCIOS EM EMPRESAS DE LINHA BRANCA
}

\section{INFLUENCES OF OPERATIONS STRATEGY IN THE SUPPLY CHAIN MANAGEMENT: AN APPROACH BASED ON BUSINESS PROCESSES IN WHITE GOOD COMPANIES}

\author{
Ana Beatriz Lopes de Sousa Jabbour ${ }^{1}$; Alceu Gomes Alves Filho ${ }^{2}$;Maria Rita Pontes Assumpção ${ }^{3}$ \\ ${ }^{1}$ Universidade Federal de São Carlos - UFSCar - São Carlos - Brasil \\ beatriz@dep.ufscar.br \\ ${ }^{2}$ Universidade Federal de São Carlos - UFSCar - São Carlos - Brasil \\ alceu@power.ufscar.br \\ ${ }^{3}$ Universidade Católica de Santos - Unisantos - Santos - Brasil \\ rita.assumpcao.@unisantos.br
}

\begin{abstract}
Resumo
Este trabalho tem como objetivo entender como a estratégia de produção de uma montadora considerada focal em sua cadeia no setor de linha branca brasileiro pode influenciar a gestão da cadeia de suprimentos mantida com seus fornecedores de primeira camada. A pesquisa é caracterizada como exploratória e qualitativa, sendo os meios de obtenção de informações as observações diretas, retenção de documentos e os dados das entrevistas realizadas com representantes de nível gerencial das áreas de compras e desenvolvimento de produtos na empresa focal e da área de produção nos fornecedores. A contribuição deste é a verificação de que a estratégia de produção da empresa focal influencia a sua gestão da cadeia de suprimentos e que a execução dos processos de negócios em comum com seus fornecedores são um meio para verificar tal afirmação. Além do mais, os fornecedores demonstram forte cooperação com a empresa focal ao complementar seus processos de negócios e com isso, apóiam-na a atender sua estratégia de produção. Foi proposto um conjunto de mecanismos para auxiliar no entendimento de como a estratégia de produção pode afetar o perfil dos processos de negócios e assim, alcançar o resultado da pesquisa.
\end{abstract}

Palavras-chaves: estratégia de produção, gestão da cadeia de suprimentos, processos de negócios.

\section{Introdução}

A desverticalização da produção tem se acentuado nos últimos anos em empresas de manufatura, em decorrência da busca por maior eficiência e redução de custos. Esse fenômeno 
contribui para tornar ainda mais complexa a coordenação das ações nas cadeias de suprimentos e reforça o desafio para os gestores na perpetuação de suas estratégias e na gestão do relacionamento com clientes e fornecedores, de modo a lidar apropriadamente com as novas exigências de mercado, tais como variação do mix de produtos, flutuação da demanda, customização de pedidos e rapidez de atendimento.

Estratégia empresarial consiste segundo Quinn (1988), num padrão ou plano que integra os objetivos, políticas e seqüência de opções num todo coeso. Os planos e ações dos dirigentes personificam a estratégia empresarial. Sendo assim, a estratégia empresarial pode ser identificada a partir das decisões implementadas. Slack et al. (2002) consideram que a função produção exerce papel estratégico na empresa, pois é ela quem suporta e implementa as atividades do sistema produtivo (de transformação), em apoio à estratégia de atuação da empresa. Assim, a estratégia de produção (EP) é o cerne de uma empresa para atingir as metas pretendidas, necessitando então de uma consistência entre conteúdo da EP e as decisões organizacionais para o sucesso daquela.

Para Ghodeswar e Vaidyanathan (2008) o processo de desverticalização e conseqüentemente, a terceirização da produção é motivada pela busca de melhorar o nível de criação de valor ao consumidor final e manter o foco em melhorias e reduções de custos. Para auferir estes ganhos, a tônica da gestão da produção das empresas foi alterada. As organizações delegam cada vez mais a produção dos componentes do produto final aos fornecedores, cabendo lhes executar as atividades de maior valor agregado e gerenciar a cadeia de organizações envolvidas nos processos de transformação. Tal abordagem de gestão da produção é denominada Gestão da Cadeia de Suprimentos (GCS).

Autores como Gattorna (1998), Seuring (2003), Chopra e Meindl (2003) e Sahay et al. (2006) comentam a necessidade da compatibilidade entre a estratégia de uma empresa e a sua GCS para atingir a estratégia pretendida. Já os trabalhos como Maia et al. (2005), Demeter et al. (2006) e Vachon et al. (2009) afirmam que a EP tem uma forte conexão com a sua CS. Considerando-se essas afirmativas e principalmente na ausência de um indicativo de como ocorrem esses relacionamentos o que motiva a realização desta pesquisa é responder "como a estratégia de produção de uma empresa focal pode influenciar a GCS mantida com seus fornecedores de primeira camada?"

O principal objetivo deste trabalho é entender como a EP de uma empresa focal pode influenciar a forma de gerenciar a cadeia de suprimentos mantida com seus fornecedores de primeira camada. Foi realizado um estudo de caso entre uma montadora do setor de linha branca e dois de seus fornecedores de componentes e a pesquisa baseou-se na formulação de um "conjunto de mecanismos" para verificar a influencia da estratégia de produção no perfil desse e assim, compreender como esse afeta a execução dos processos de negócios entre a montadora e seus 
fornecedores. Lembrando que segundo a literatura de GCS a execução dos processos de negócios indicam como a GCS pode ser implementada.

Este trabalho está estruturado em seções: 2 - aspectos da literatura, 3 - método de pesquisa, 4 - estudo de caso, 5 - principais resultados e 6 - considerações finais.

\section{Aspectos da Literatura}

O tema estratégia empresarial é discutido vastamente na literatura, apresentando várias definições, tais como: (a) um plano de ação (SKINNER, 1969), (b) conjunto de valores organizacionais (HAYES e WHEELWHRIGHT, 1984) e (c) um posicionamento de mercado (SLACK et al., 2002). A revisão bibliográfica adotada neste trabalho segue a vertente tradicional, uma perspectiva top down, ou seja, a estratégia é definida pelos interesses da empresa, onde ela quer chegar. Nessa perspectiva, a estratégia possui três níveis de divisão, corporativa, negócios e funcionais, cada qual tendo a responsabilidade por um conjunto de decisões distintas, mas ligadas hierarquicamente. A EP, segmento da estratégia funcional, é foco das discussões do trabalho, uma vez que ela, segundo Fine e Hax (1985) é uma parte crítica da estratégia corporativa e de negócios, compreendendo um conjunto bem coordenado de objetivos e programas de ação, objetivando assegurar, a longo prazo, vantagem competitiva da empresa.

Os principais elementos que identificam a EP são as áreas estruturais e infra-estruturais e as prioridades competitivas da função produção. Segundo Hayes e Wheelwright (1984) e Hayes et al. (1988), entende-se por decisões na área estrutural de produção aquelas de impacto de longo prazo e que requerem substancial investimento de capital, incluindo escolhas nas seguintes áreas: (a) capacidade de produção (quantidade produzida, forma de produção); (b) instalações (localização, tamanho, layout); (c) tecnologia (tipo de equipamento, tipo de processo); e (d) integração vertical (a extensão do relacionamento com os fornecedores). Por seu turno, as decisões infra-estruturais são aquelas de curto prazo relacionadas a aspectos de gestão; distinguem-se entre essas decisões: (a) capital intelectual (habilidades dos colaboradores, educação, políticas de contratação); (b) programas de qualidade (prevenção a defeitos, monitoramento, intervenção); (c) planejamento da produção/controle de materiais (políticas de aquisição, critérios de decisão, centralização); (d) organização (estrutura, sistema de desempenho, o papel dos líderes) e (e) desenvolvimento de novos produtos (compartilhar o desenvolvimento com fornecedores e clientes?; ou centralizar os aspetos de projeto do produto?). As prioridades competitivas definem a forma como a empresa pretende competir no mercado para atender as necessidades de seus clientes e são segundo Garvin (1993): custo, qualidade, entrega, flexibilidade e serviço. A partir de uma pesquisa bibliográfica sobre o tema, Leong et al. (1990) apontam as seguintes definições para esses objetivos: 
a) Custo: produção e distribuição de produtos a baixo preço;

b) Qualidade: produção de produtos com alta qualidade e padrão de desempenho;

c) Entrega: disponibilidade e velocidade de entrega;

d) Flexibilidade: mix de produto e volume;

e) Serviço: suporte no atendimento ao cliente.

Obs: Na pesquisa de campo, considerou a prioridade "serviço" também com uma perspectiva de apoio ao fornecedor, prática comum numa GCS.

Voss (2005) observou diferentes escolhas e maneiras das empresas competirem no mercado e constatou que o sucesso e o fracasso das empresas podiam ser explicados pelas escolhas (áreas de decisão e prioridades competitivas) feitas e o alinhamento dessas escolhas com a estratégia competitiva. Uma forma de conseguir esta compatibilidade é partir das inter-relações funcionais, uma que vez que as interfaces entre a função produção e as demais contribuem para que todas as funções e pessoas foquem suas atividades no mesmo objetivo.

Se por um lado o tema estratégia é vastamente debatido na literatura há anos, o tema GCS, motivador desta pesquisa, é um assunto ainda recente. Na década de 1980 despertou o interesse da academia e hoje desponta como um tema chave para entender a gestão da produção.

Segundo Christopher (1998), cadeia de suprimentos (CS) é uma rede de organizações que estão envolvidas por meio de ligações a jusante (lado dos clientes) e a montante (lado dos fornecedores) nos diferentes processos e atividades que produzem valor na forma de produtos e serviços liberados ao consumidor final. Gerir esses processos e a rede é um dos desafios correntes das organizações, pois segundo Fisher (1997) cada tipo de produto requer uma estrutura de CS que suporte as operações necessárias para atender ao perfil do cliente daquele produto. Ou seja, se o produto é funcional (demanda estável) as características da estrutura e da gestão da CS são voltadas a redução de custos, se o produto é inovador (demanda variável) espera-se que a CS opere de forma ágil para atender as demandas flutuantes.

A lógica da GCS alude à ótica defendida por Prahalad e Hamel (1990), ou seja, as empresas enfatizam suas competências internas requerendo maior ligação com fornecedores para apoio às atividades não centrais do sistema produtivo da empresa focal. Algumas definições para GCS são: (a) integração dos processos de negócios desde o usuário final até os fornecedores originais, para proporcionar produtos, serviços e informações que agregam valor ao cliente (COOPER, LAMBERT e PAGH, 1997) e (b) é a filosofia que estende as tradicionais atividades internas de uma empresa pelo envolvimento do escopo inter-organzacional, por meio da integração com os parceiros com metas comuns de otimização e eficiência (HARWICK, 1997). 
Uma série de mudanças comportamentais é apontada para que as empresas se adéqüem a uma GCS para obter os ganhos da gestão dos relacionamentos, destacando-se a maior troca de informações entre cliente-fornecedor, o estabelecimento de metas comuns, a integração de processos, a construção de relacionamento de longo prazo e a cooperação na busca pela eficiência conjunta (MENTZER et al., 2001).

Para Lambert e Cooper (2000) o sucesso de uma GCS requer a mudança da gestão individual das funções para a integração das atividades dentro dos processos de CS. Portanto, esse assunto é destacado neste trabalho. The Global Supply Chain Forum (GSCF) em uma das pesquisas com seus afiliados constatou que existem 8 tipos de processos de negócios entre empresas de uma CS (CROXTON et al. 2001). Esses processos são:

(a) Gestão do relacionamento com o cliente - são os processos que fornecem a estrutura de como o relacionamento com os clientes é desenvolvido e mantido.

(b) Gestão de serviço ao cliente - esse processo serve para monitorar o desempenho da empresa e o nível de satisfação do cliente.

(c) Gestão da demanda - esse processo busca balancear os requisitos do cliente com a capacidade de fornecimento da empresa e sincronizar a previsão de demanda com a produção, aquisição e distribuição.

(d) Atendimento ao pedido do cliente - esse processo busca atender as necessidades discriminadas pelo cliente.

(e) Gestão do fluxo de produção - esse processo é responsável pela produção dos produtos demandados pelos clientes e o estabelecimento da flexibilidade necessária para atender os mercados alvo.

(f) Gestão do relacionamento com o fornecedor - é o processo que define a forma como a empresa interage com seus fornecedores para obter assertividade dos produtos e serviços comprados.

(g) Desenvolvimento de produto e comercialização - o processo de desenvolver produtos considera as informações advindas dos processos de gerenciamento do relacionamento com o cliente (necessidades dos clientes), do gerenciamento do relacionamento com os fornecedores (seleção de materiais e fornecedores) e fluxo de produção (processo produtivo).

(h) Gestão do retorno - oferece algumas oportunidades de alcançar a vantagem competitiva sustentável, a partir da gestão reversa de sua CS.

A Figura 1 apresenta os processos de negócios descritos.

Lambert e Cooper (2000) indicam que a implementação da GCS envolve identificar membros da CS com quem se mantém transações críticas, quais processos de negócios estão envolvidas nessas transações e que nível de integração é aplicado a esses processos. Em função 
desta orientação, elegeu-se "um conjunto de mecanismos" adotado pelas áreas funcionais das organizações para ajudar a verificar a integração que ocorre entre processos de negócios de empresas numa CS. Neste trabalho entende-se por "conjunto de mecanismos" algumas atividades desenvolvidas em uma organização para facilitar a integração entre os processos de cliente e fornecedor. As atividades escolhidas são: seleção de fornecedores, relacionamento clientefornecedor, troca de informações e early supplier Involvement (ESI).

Figura 1: Processos de negócios ao longo de uma CS.

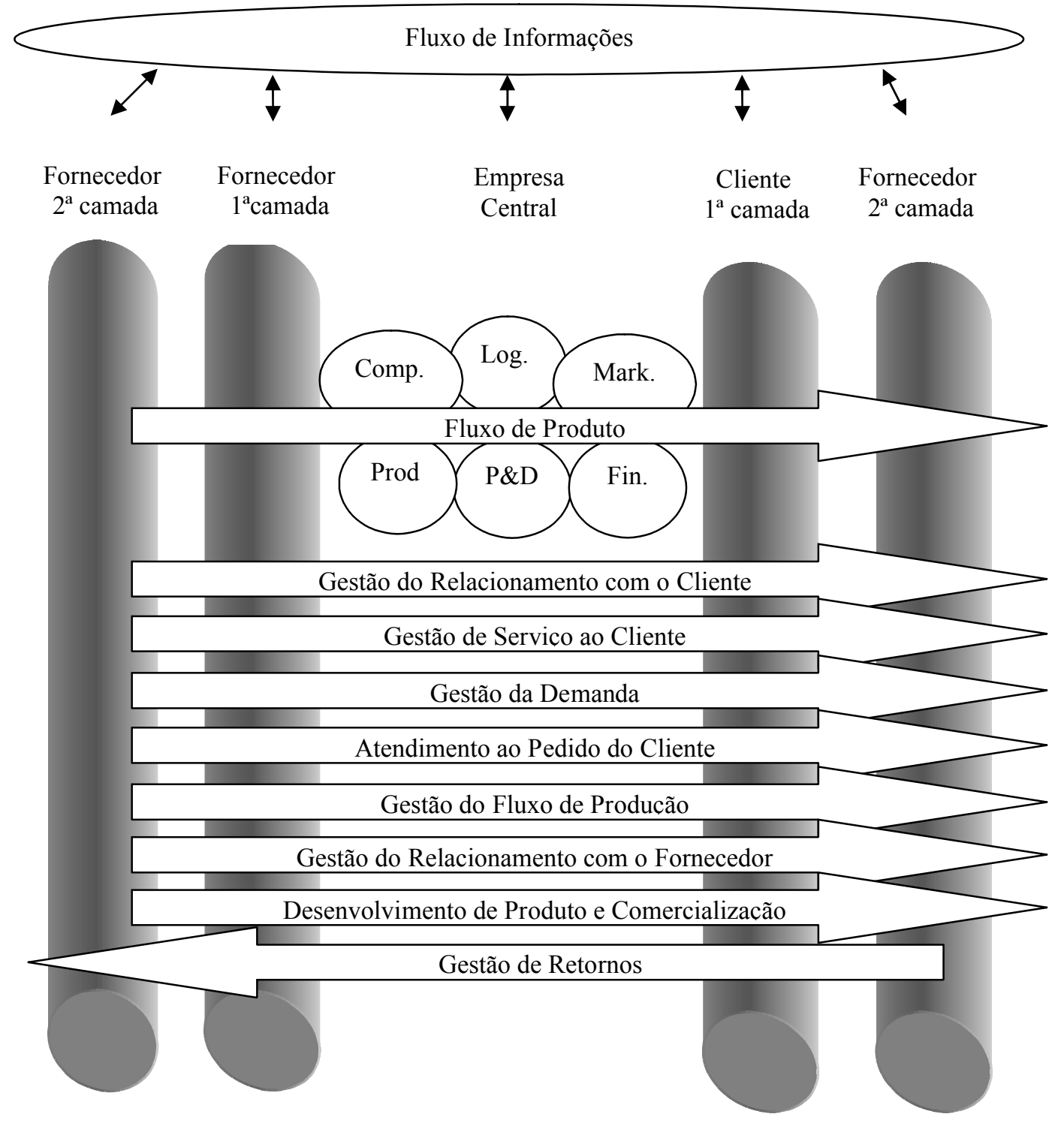

Fonte: Croxton et al. (2001).

A seleção de fornecedores representa decidir quais organizações podem ser consideradas aptas a prover suprimentos a uma empresa. Para tanto, quando existem amplas opções no mercado, como organizações de grande, médio e pequeno porte; tradicionais ou novos entrantes; avançadas 
tecnologicamente ou tecnologicamente maduras, enfim, a função compras necessita estabelecer critérios de seleção. Para Furtado (2005) os critérios têm sido cada vez mais amplos e subjetivos, pois se busca não apenas um fornecedor, mas um parceiro para manter relações de longo prazo e com o qual se possa compartilhar objetivos e promover sinergias entre as estratégias de ambas as empresas.

Assim como vêm ocorrendo mudanças nos critérios de seleção de fornecedores nos últimos anos, a forma de se relacionar com eles também vem se alterando, dado o maior comprometimento necessário na relação estabelecida em função da desverticalização da cadeia produtiva. Antigamente, a função compras considerava a relação cliente-fornecedor como meramente comercial, sendo os fornecedores vistos como pontos de venda onde se comprava pelo melhor preço. Claro et al. (2006) confirmam que o fornecedor tem sido cada vez mais importante na obtenção da vantagem competitiva de seus parceiros da CS. Fornecedores chaves estão participando dos projetos de desenvolvimento de novos produtos, a exemplo da indústria automobilística (LEEMORTIMER, 1994). Estes fornecedores são responsáveis pela produção de componentes específicos a um produto do cliente e para isso compartilham informações com o membro focal da cadeia para serem capazes de responder as demandas desse.

Uma importante característica na relação cliente-fornecedor é a quantidade e o tipo de informações compartilhadas entre os membros de uma CS (LI et al., 2006). Para Barratt (2004) a troca de informações tem sido um pré-requisito para a colaboração numa CS em virtude do impacto destas no desempenho.

A atividade de desenvolvimento de novos produtos tem se caracterizado como uma das mais centrais no âmbito da conquista da vantagem competitiva (BIROU e FAWCETT, 1994). Uma prática que auxilia a redução do lead time de desenvolvimento de produtos é a prévia participação de fornecedores nas etapas iniciais (ESI) dessa atividade. O envolvimento de fornecedores refere-se aos recursos (capacidade, investimentos, informação, conhecimento, idéias) que eles disponibilizam, as tarefas que realizam, as responsabilidades que eles assumem em relação ao desenvolvimento de uma parte, processo, ou serviço para beneficiar os projetos desenvolvidos atuais e futuros dos clientes (VAN ECHTELT et al., 2006).

Esta seção procurou elucidar, a partir de elementos da literatura, a forma encontrada de verificar a relação entre EP e GCS.

\section{Método de Pesquisa}

Este trabalho pretende entender como a EP de uma empresa focal pode influenciar a forma de gerenciar a cadeia de suprimentos estabelecida com fornecedores de primeira camada. Sabe-se, 
que pesquisas como a de Maia et al. (2005) obtiveram o resultado de que EP abrange em suas áreas de decisão uma série de aspectos que são intimamente ligados a GCS. Questões como o nível de integração vertical, o planejamento e controle da produção, o desenvolvimento de produtos, entre outras são fortemente influenciadas (ao mesmo tempo em que influenciam) por aspectos estruturais e relacionais da GCS. E a pesquisa de Vachon et al. (2009) conclui que o alinhamento entre a EP e GCS ocorre por meio do tipo de relacionamento mantido com os fornecedores. No entanto, não há um modelo de referência já consolidado para análise do relacionamento proposto pela pesquisa. Cerra et al. (2007) indicam como proposta de pesquisa futura detalhar como elementos operacionais se combinam para apoiar as estratégias competitivas e de GCS das empresas. Em função disso, o método de pesquisa escolhido para este trabalho foi o estudo de caso. O método de estudo de caso é utilizado quando a pesquisa tenta elucidar questões do tipo "como algo ocorre" ou "por que está acontecendo algo". Para Voss et al. (2002) o estudo de caso é um método de pesquisa particularmente consistente para o desenvolvimento de novas teorias. Ele é destinado tanto para elementos físicos, como humanos da organização. Para Yin (2004), as principais características desse método são: estuda-se o fenômeno no seu ambiente natural, a investigação é exploratória, pois as variáveis ainda são desconhecidas, e o fenômeno não é totalmente compreendido.

Por se tratar de um estudo exploratório e as variáveis ainda não serem totalmente conhecidas, parte-se, então de pressupostos como: (a) o "conjunto de mecanismos" ajuda a identificar a forma como EP interfere na execução dos processos de negócios e (b) a execução dos processos de negócios ajuda a entender como a GCS de uma empresa focal pode ser implementada. Estas afirmativas foram desenvolvidas a partir de alguns elementos da literatura. Primeiro pela perspectiva top down da estratégia, Voss (2005) constatou que o sucesso e o fracasso das empresas podiam ser explicados pelas escolhas (áreas de decisão e prioridades competitivas) feitas e o alinhamento dessas escolhas com a estratégia competitiva. Uma forma de conseguir esta compatibilidade é partir das inter-relações funcionais, uma que vez que as interfaces contribuem para que todas as funções e pessoas foquem suas atividades no mesmo objetivo. Dessa maneira, o "conjunto de mecanismos", que são atividades realizadas por áreas funcionais como Compras e Desenvolvimento de Produto, apóia a EP da empresa para que esta se concretize. Sendo assim, este "conjunto de mecanismos", possui uma abordagem que se assemelha ao perfil da EP. A partir da definição do "conjunto de mecanismos" é possível identificar como os processos de negócios são realizados, pois segundo Lambert e Cooper (2000) processos de negócios é a integração das atividades dentro dos processos de CS. O segundo pressuposto deriva da afirmação de Lambert e Cooper (2000) que indicam que a implementação da GCS envolve identificar membros da CS com quem se mantém transações críticas, quais processos de negócios estão envolvidas nessas transações e que nível de integração é aplicado a esses processos. 
A Figura 2 ilustra a lógica que une os pressupostos assinalados. A EP da empresa focal norteia o formato do conjunto de mecanismos e esses são como diretrizes para os fornecedores de primeira camada na execução dos processos de negócios em comum com a focal. A forma como os fornecedores executam os processos de negócios podem afetar sua gestão interna (mudanças organizacionais) e isso permite verificar como a EP afeta aspectos de GCS.

Figura 2: Conjunto de relacionamentos dos pressupostos considerados.

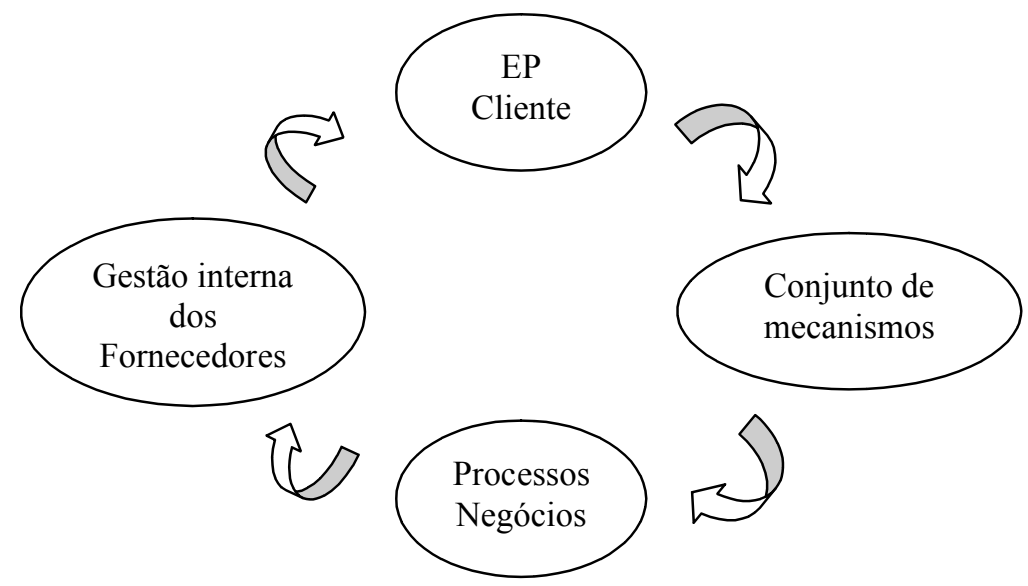

Fonte: Autoria própria (2007).

Três recortes foram definidos em termos de escopo desta pesquisa: (a) são analisados neste trabalho dois elos a montante da cadeia de suprimentos da montadora (atual líder brasileira no setor de linha branca), sendo essa chamada como empresa focal em função da delimitação da pesquisa (uma montadora e dois fornecedores), (b) o conjunto de mecanismos foi determinado dado o recorte da pesquisa e em função dos insights da pesquisa de Maia et. al. que afirmam que as áreas relacionadas de integração vertical (seleção de fornecedores e relacionamento cliente-fornecedor), planejamento e controle da produção (troca de informações) e desenvolvimento de produtos (ESI) estão intimamente ligados a GCS e (c) analisam-se os processos de negócios relacionados ao elo montadora e fornecedor. Dos processos de negócios do modelo de Lambert e Cooper (2000) e Croxton et al. (2001) para GCS, são considerados neste estudo: gestão da demanda, gestão do fluxo de produção, gestão do relacionamento com o fornecedor e desenvolvimento de produto e comercialização. A justificativa para a escolha desses processos é em função da importância dos fornecedores quanto às questões: (1) resposta rápida às variações da demanda, (2) execução de operações delegadas pela montadora, que se referem, em parte, às decisões estruturais e infraestruturais daquela, (3) confiabilidade dos produtos e (4) desenvolvimento de soluções tecnológicas para novos produtos

Dois fornecedores foram escolhidos para participar desta pesquisa, selecionados em razão do teor crítico do insumo no desempenho do produto final e pela peculiaridade do insumo. Um deles 
possui como característica a fácil coordenação com a gestão da montadora; o outro é caracterizado pela forte integração no processo de desenvolvimento de produto da montadora.

Este trabalho se desenvolveu em quatro etapas: (a) fundamentação teórica, (b) elaboração de roteiros de entrevista, (c) realização do estudo de caso e (d) análise dos resultados e dissertação das principais considerações a respeito das descobertas. A partir da fundamentação teórica realizada, os roteiros de entrevista foram preparados no intuito de identificar e caracterizar os elementos que constituem a EP e a GCS dos objetos de estudo, montadora e fornecedores de primeira camada. $\mathrm{O}$ conteúdo dos roteiros seguiu três perspectivas: (1) a partir das necessidades dos clientes da montadora, como se caracteriza a sua EP?; (2) quais são os elementos da EP da montadora que influenciam seus processos de negócios?; (3) como os fornecedores são afetados pela EP da montadora na execução de seus dos processos de negócios? Os entrevistados na montadora foram funcionários de nível gerencial das áreas de desenvolvimento de produtos e compras. Em busca de maior validade das informações obtidas na montadora, dois fornecedores chaves também foram entrevistados para verificar as configurações das suas inserções na GCS da montadora. Os entrevistados junto às empresas fornecedoras compõem o nível gerencial da área de produção nessas unidades. Em cada empresa foram realizadas duas visitas de duração de cerca em três horas cada que possibilitaram observações diretas as linhas de produção e as áreas administrativas e retenção de documentos não confidenciais. Nos dois momentos foram realizadas entrevistas com as pessoas indicadas, citadas anteriormente. O roteiro de entrevistas foi seguido plenamente e as entrevistas tiveram duração de cerca de duas horas, não foi possível gravar as respostas. E a pesquisa de campo foi desenvolvida ao longo de dois anos (2005 a 2007).

Após a realização das visitas às empresas e suas conseqüentes coletas dos dados, as análises e reflexões (comparação entre teoria e prática) foram desenvolvidas para conclusão da pesquisa.

\section{Estudo de Caso}

Três empresas do setor de linha branca foram selecionadas para este trabalho: uma montadora de grande porte e líder do setor e dois de seus fornecedores de componentes eletroeletrônicos. O setor de linha branca foi escolhido para realizar esta pesquisa em função de seu comportamento, em termos de gestão, pois este busca se semelhar a gestão do setor automotivo, setor que alavanca as mudanças na área de gestão de operações.

Por questões de confidencialidade os nomes das empresas foram resguardados e são intituladas como: Alfa, a montadora, e Beta e Gama, os fornecedores.

\section{Empresa Alfa}




\section{Características de Alfa}

Alfa é uma montadora de grande porte do setor de linha branca no Brasil. Os principais produtos produzidos são: lavadora, refrigerador, freezer, fogão, microondas e lava louça. Essa está localizada no Estado de São Paulo no Brasil e pertence a um grupo americano que atua em negócios diversificados.

Alfa adota uma estratégia de segmentação de clientes via marcas diferentes para seus produtos. A Marca 1 atende aos clientes finais de classe A (alto poder aquisitivo) e a Marca 2 é direcionada às necessidades de clientes finais de classe $\mathrm{B}$ e $\mathrm{C}$ (médio poder aquisitivo). Por se tratar de segmentos diferentes de mercado, Alfa foca em distintos esforços de desempenho para atendêlos. Para a Marca 1 as prioridades são qualidade e inovação e para a Marca 2 qualidade e custos.

Desde a chegada ao Brasil das grandes empresas do mercado global, a partir da metade da década de 1990, o setor de linha branca vem se tornando mais competitivo, acirrando a disputa por preço, uma vez que a qualidade é considerada critério qualificador de pedidos. Aspectos ligados ao posicionamento da empresa Alfa quanto a custo e diferenciação oscilaram nos último sete anos, na busca de atender as demandas emergentes dos clientes. O Quadro 1 ilustra esta variação.

Quadro 1: Análise comparativa do posicionamento de Alfa quanto à importância dos critérios custo e diferenciação

(2002 e 2007)

\begin{tabular}{l|c|c}
\hline \multicolumn{1}{c|}{$\begin{array}{c}\text { Posicionamento } \\
\text { Característica focada }\end{array}$} & $\mathbf{2 0 0 7}$ & $\mathbf{2 0 0 2}$ \\
\hline CUSTO & 1 & 2 \\
\hline Redução de custos & 1 & 2 \\
\hline Obtenção do menor custo & 2 & 3 \\
\hline Obtenção do menor preço de venda & & 1 \\
\hline DIFERENCIAÇÃO & 1 & 3 \\
\hline Diferenciação de produto & 2 & 1 \\
\hline Diferenciação da entrega e serviço & 1 & 2 \\
\hline Diferenciação da qualidade & 1 & 1 \\
\hline Diferenciação por inovação & 1 & \\
\hline Diferenciação da marca & & \\
\hline
\end{tabular}

\begin{tabular}{|l|l|}
\hline \multicolumn{1}{|c|}{ Escala } & \multicolumn{1}{c|}{ Intensidade } \\
\hline 1 & Muito importante \\
\hline 2 & Importante \\
\hline 3 & Pouco importante \\
\hline
\end{tabular}

Fonte: Pesquisa de campo (2007).

Alfa busca reduzir custos principalmente os relacionados às operações internas (interferência externa - fornecedores) e investe cada vez mais em inovação de seus produtos e processos. Em parte, este panorama está correlacionado com a sua estratégia de segmentação (Marca 1 - inovação, Marca 2 - custo), refletindo em suas prioridades competitivas (Quadro 2). 
Quadro 2: Evolução da percepção das prioridades competitivas de Alfa (2002 a 2007).

\begin{tabular}{|c|c|c|}
\hline $\begin{array}{l}\text { Prioridades Competitivas } \\
\end{array}$ & 2007 & 2002 \\
\hline \multicolumn{3}{|l|}{ CUSTO } \\
\hline $\begin{array}{l}\text { Custo de produção (custo total do } \\
\text { produto vendido) }\end{array}$ & 1 & 2 \\
\hline Custo com mão-de-obra & 1 & 2 \\
\hline Custo com insumos & 1 & 1 \\
\hline \multicolumn{3}{|l|}{ QUALIDADE } \\
\hline Conformidade com o projeto & 1 & 1 \\
\hline Prevenção de falhas & 1 & 1 \\
\hline Rastreabilidade/controle dos processos & 1 & 1 \\
\hline \multicolumn{3}{|l|}{ FLEXIBILIDADE } \\
\hline $\begin{array}{l}\text { Flexibilidade para atender as variações } \\
\text { de mix de produtos na linha de } \\
\text { produção }\end{array}$ & 1 & 2 \\
\hline $\begin{array}{l}\text { Flexibilidade para responder as } \\
\text { variações de quantidade de lotes de } \\
\text { pedidos }\end{array}$ & 2 & 3 \\
\hline $\begin{array}{l}\text { Flexibilidade de inovação em processos } \\
\text { ou produtos }\end{array}$ & 1 & 2 \\
\hline \multicolumn{3}{|l|}{ SERVIÇO } \\
\hline $\begin{array}{l}\text { Apoio aos clientes no desenvolvimento } \\
\text { de novos produtos }\end{array}$ & 2 & 2 \\
\hline Resolução de problemas & 1 & 2 \\
\hline Apoio ao fornecedor & 1 & 2 \\
\hline \multicolumn{3}{|l|}{ ENTREGA } \\
\hline $\begin{array}{l}\text { Rapidez de atendimento ao pedido do } \\
\text { cliente }\end{array}$ & 2 & 2 \\
\hline $\begin{array}{l}\text { Cumprimento com os prazos e } \\
\text { especificações de entrega }\end{array}$ & 2 & 2 \\
\hline
\end{tabular}

\begin{tabular}{|l|l|}
\hline \multicolumn{1}{|c|}{ Escala } & \multicolumn{1}{c|}{ Intensidade } \\
\hline 1 & Muito importante \\
\hline 2 & Importante \\
\hline 3 & Pouco importante \\
\hline
\end{tabular}

Fonte: Pesquisa de campo (2007)

A transformação mais significativa que vem se evidenciando nas operações de Alfa é o foco em redução de custos de produção, o aumento da flexibilidade para diferenciação dos produtos e o apoio aos fornecedores na resolução de problemas. Essas prioridades relacionam-se diretamente tanto com a demanda dos diferentes segmentos dos clientes finais, como com as exigências dos clientes intermediários, os varejistas. As margens de lucro do setor de linha branca são baixas, então uma forma de aumentar participação de Alfa no varejo é a partir de mix de produtos.

Uma maneira de Alfa atingir esses objetivos de desempenho é, principalmente, a partir de um relacionamento mais próximo com os fornecedores. Esses são envolvidos em programas de qualidade em níveis de projeto de componente e de processos de fabricação. Há também práticas de ESI com programa formal de interação de equipes de projeto interorganizacionais. Isso facilita a redução de custos na fase de projeto, por evitar retrabalhos, uma vez que essa fase é interativa e facilita a flexibilidade na inovação. 
O mercado de linha branca possui duas peculiaridades que influenciam a gestão da produção das montadoras: sazonalidade na demanda e poder de barganha do varejo na CS. A demanda é regida pelos principais varejistas do mercado, os grandes magazines e hiper-mercados. Eles fazem os pedidos apenas nos últimos dias do mês, como conseqüência, Alfa é obrigada a acumular estoque ao longo do mês. Assim, Alfa necessita de capacidade de gestão de seu mix de produtos acabados, pois ela trabalha com uma demanda independente que pode acarretar em custos de estoque ou risco de não entrega ao cliente. Nesse sentido, muitos fornecedores de Alfa se tornam essenciais para seu melhor desempenho. Pode-se constatar isso a seguir, a partir da descrição da execução de alguns processos de negócios de Alfa, com os quais a montadora complementa sua capacitação na gestão de sua capacidade produtiva.

Quadro 3: Caracterização dos processos de negócios de Alfa

\begin{tabular}{|c|c|c|}
\hline Processos de Negócios & Características & Ligação com a EP de Alfa \\
\hline $\begin{array}{l}\text { Gestão do Fluxo de Produção } \\
\text { (GFP) }\end{array}$ & $\begin{array}{l}\text { Cálculo das necessidades de } \\
\text { materiais via sistema MRP. } \\
\text { Itens programados por kanban e } \\
\text { coletas por milk run. }\end{array}$ & Redução de custos com estoque. \\
\hline Gestão da Demanda (GD) & $\begin{array}{l}\text { Troca de informações por um } \\
\text { portal eletrônico. } \\
\text { Disponibilidade de informações de } \\
\text { previsão de demanda e pedidos de } \\
\text { compra. }\end{array}$ & $\begin{array}{l}\text { Apoio aos fornecedores para que } \\
\text { eles possam se programar com } \\
\text { antecedência e assim atender as } \\
\text { demandas reais de Alfa, havendo } \\
\text { uma redução nos custos de } \\
\text { estoque de matéria-prima. }\end{array}$ \\
\hline $\begin{array}{c}\text { Gestão do Relacionamento com } \\
\text { o Fornecedor (GRF) }\end{array}$ & $\begin{array}{l}\text { Os fornecedores são selecionados } \\
\text { principalmente por critérios } \\
\text { como: qualidade, capacidade } \\
\text { produtiva, custos de fornecimento e } \\
\text { capacidade de inovação. } \\
\text { A empresa usa a política de dual } \\
\text { source. } \\
\text { Os fornecedores estratégicos } \\
\text { participam e trocam informações } \\
\text { sobre desenvolvimento de novos } \\
\text { produtos. }\end{array}$ & $\begin{array}{l}\text { Apoio aos fornecedores para que } \\
\text { eles possam colaborar nos } \\
\text { processos da montadora e na } \\
\text { busca de inovação. }\end{array}$ \\
\hline $\begin{array}{l}\text { Desenvolvimento de Produto } \\
\text { (DP) }\end{array}$ & $\begin{array}{l}\text { Alfa adota a prática do ESI para } \\
\text { obter a contribuição do know how } \\
\text { de alguns de seus fornecedores em } \\
\text { novos produtos. }\end{array}$ & $\begin{array}{l}\text { Busca de inovação de produto e } \\
\text { de processo e redução de custos } \\
\text { em virtude do menor tempo de } \\
\text { time to market. }\end{array}$ \\
\hline
\end{tabular}

Fonte: Pesquisa de campo (2007)

Percebe-se pelas informações contidas no Quadro 1 que o conjunto de mecanismos, seleção de fornecedores, relacionamento com fornecedores, troca de informações e a prática do ESI são vetores para a execução dos processos de negócios de Alfa. Além disso, a execução dos processos de negócios apóia que a EP de Alfa possa ser concretizada. 


\section{Empresa Beta}

Beta é uma montadora de grande porte de componentes elétricos e eletrônicos que atende o setor automobilístico e o de linha branca. Fornece produtos como chicotes elétricos, cordões de força e placas eletrônicas. Ela possui duas unidades produtivas, uma delas é estudada neste trabalho, a localizada no Estado de São Paulo.

A parceria entre Alfa e Beta já perdura por mais de 13 anos. Os itens fornecidos por Beta são específicos a cada mix de produto de Alfa, mesmo sendo considerados commodities com baixo conteúdo tecnológico envolvido em seus processos. Alfa corresponde a $50 \%$ do faturamento de Beta com o setor de linha branca. A forma como Beta complementa os processos de negócios de Alfa são ilustrados no quadro 4.

Quadro 4: Caracterização dos processos de negócios de Beta.

\begin{tabular}{|c|c|c|}
\hline Processo de Negócio & Caracterização & Relação com Alfa \\
\hline $\begin{array}{l}\text { Gestão do Fluxo de Produção } \\
\text { (GFP) }\end{array}$ & $\begin{array}{l}\text { Cálculo das necessidades de } \\
\text { materiais via sistema MRP. } \\
\text { Entregas acionadas por sistema } \\
\text { kanban. }\end{array}$ & $\begin{array}{l}\text { As entregas por sistema kanban } \\
\text { se iniciaram em virtude das } \\
\text { necessidades de Alfa. Dessa } \\
\text { forma, Beta conseguiria } \\
\text { compatibilidade de capacidade } \\
\text { produtiva, um dos critérios de } \\
\text { seleção daquela. Além disso, } \\
\text { Alfa consegue reduzir custos } \\
\text { com estoque. }\end{array}$ \\
\hline Gestão da Demanda (GD) & $\begin{array}{l}\text { Beta implementou um software } \\
\text { chamado Neogrid para facilitar a } \\
\text { troca de informações com Alfa. } \\
\text { Beta obtém informações de } \\
\text { previsão de demanda e nível de } \\
\text { estoque de seus itens na planta } \\
\text { de Alfa a partir do Neogrid. }\end{array}$ & $\begin{array}{l}\text { O Neogrid permite maior } \\
\text { integração entre Alfa e Beta, o } \\
\text { que facilita o relacionamento } \\
\text { entre eles. A partir da troca de } \\
\text { informações sobre materiais, } \\
\text { Beta consegue se programar } \\
\text { melhor e assim atender as reais } \\
\text { necessidades de Alfa. }\end{array}$ \\
\hline $\begin{array}{l}\text { Desenvolvimento de Produto } \\
\text { (DP) }\end{array}$ & $\begin{array}{l}\text { Beta desenvolveu uma equipe de } \\
\text { engenheiros de aplicação para } \\
\text { viabilizar novos projetos de } \\
\text { produtos para Alfa. }\end{array}$ & $\begin{array}{l}\text { A partir de uma equipe focada } \\
\text { nas necessidades de produtos de } \\
\text { Alfa, essa consegue obter } \\
\text { inovação. Ou seja, com a } \\
\text { prática do ESI, Alfa atinge um } \\
\text { de seus objetivos de } \\
\text { desempenho. }\end{array}$ \\
\hline
\end{tabular}

Fonte: Pesquisa de campo (2007).

Beta se reestruturou em alguns aspectos para atender aos requisitos de ao seu principal cliente, Alfa, e assim, complementar os processos de negócios em comum. Beta teve que se adaptar a programação da produção puxada pelo mecanismo do cartão kanban da filosofia JIT, pois as entregas passaram a ser várias vezes ao dia, enquanto antes era uma vez ao dia. O uso do software Neogrid implicou na mudança da rotina da equipe de planejamento, a qual se baseava em programação histórica da demanda e passou a ter que rever a programação diariamente pelo site, 
assim, a programação se tornou mais acurada. E por último, em função do foco de Alfa no desenvolvimento de novos produtos, Beta definiu uma equipe de engenheiros de aplicação para trabalhar direcionada as demandas de produtos de Alfa.

A atuação de Beta quanto a execução dos processos de negócios comuns a Alfa está condicionada as características do conjunto de mecanismos.

\section{Empresa Gama}

A montadora Gama é mundialmente reconhecida no fornecimento de controles e componentes eletroeletrônicos para os segmentos de linha branca, automobilístico e eletrônica. Ela está situada no Estado de São Paulo no Brasil e fornece componentes como termostato, válvula de entrada de água, módulo tracionador, timer e interruptores.

A política de Gama é integrar a produção internamente, ou seja, ela apenas adquire a matéria-prima básica e os processamentos necessários são todos realizados internamente, inclusive, a confecção de moldes para as máquinas de injeção.

A característica fundamental de Gama é a proatividade na busca de novos desenvolvimentos de produtos. Os seus produtos possuem conceito e tecnologia de processo que os tornam diferenciados e patenteáveis. Alfa é um dos principais clientes de Gama, e a parceria está cada vez mais sólida em virtude das soluções oferecidas por Gama na busca do melhor desempenho das lavadoras daquela. A seguir a descrição de seus processos de negócios (quadro 5).

Quadro 5: Caracterização dos processos de negócios de Gama.

\begin{tabular}{|c|l|l|}
\hline Processos de Negócios & \multicolumn{1}{|c|}{ Características } & \multicolumn{1}{c|}{ Relação com Alfa } \\
\hline $\begin{array}{c}\text { Gestão do Fluxo de Produção } \\
\text { (GFP) }\end{array}$ & $\begin{array}{l}\text { Cálculo das necessidades de } \\
\text { materiais via sistema MRP. } \\
\text { Entregas acionadas por sistema } \\
\text { kanban para atender Alfa. }\end{array}$ & $\begin{array}{l}\text { Para atender as exigências de } \\
\text { Alfa, Gama entrega materiais } \\
\text { pelo sistema kanban. Isso resulta } \\
\text { numa redução de estoque para } \\
\text { Alfa. }\end{array}$ \\
\hline Gestão da Demanda (GD) & $\begin{array}{l}\text { Gama mantém uma política de } \\
\text { estoque de 15 dias para todos os } \\
\text { seus clientes. }\end{array}$ & $\begin{array}{l}\text { Mesmo Gama tendo acesso no } \\
\text { portal da Internet a uma previsão } \\
\text { de demanda dos itens de Alfa, } \\
\text { ela prefere manter estoque para } \\
\text { ter a disponibilidade de } \\
\text { atendimento. }\end{array}$ \\
\hline $\begin{array}{l}\text { Desenvolvimento de Produto } \\
\text { (DP) }\end{array}$ & $\begin{array}{l}\text { Equipe de engenheiros de } \\
\text { aplicação própria para } \\
\text { desenvolver novas soluções em } \\
\text { produtos para todos seus } \\
\text { clientes. }\end{array}$ & $\begin{array}{l}\text { Gama mantém uma equipe de } \\
\text { engenheiros focados para } \\
\text { atender as demandas de Alfa. } \\
\text { Assim, Alfa consegue obter } \\
\text { inovação em produtos com a } \\
\text { prática do ESI. }\end{array}$ \\
\hline
\end{tabular}

Fonte: Pesquisa de campo (2007). 
Gama não necessitou de mudanças radicais em seus processos gerenciais para atender as necessidades de Alfa, uma explicação para isso é o fato de Gama se enquadrar no perfil das atividades do conjunto de mecanismos traçados por Alfa.

\section{Principais Resultados}

A EP de Alfa é baseada em três pilares distinguidos segundo a tendência de suas prioridades competitivas: redução de custos, flexibilidade para inovação e serviço, no que tange o apoio aos fornecedores. Essa afeta o conjunto de mecanismos, facilitadores no processo de gerenciar certos aspectos da GCS, uma vez que são considerados atividades funcionais (e inter-funcionais) da empresa. $\mathrm{O}$ conjunto de mecanismos atua como diretriz na forma que a empresa pretende executar seus processos de negócios em comum com atores da cadeia, e de certa forma, reflete sua própria estratégia.

O perfil da EP influencia diretamente o conjunto de mecanismos. Por exemplo, o foco de atuação de Alfa para atender a marca 1 é em inovação de produtos, então ela seleciona fornecedores que possam contribuir no processo de desenvolvimento de produtos, mantendo com eles um relacionamento mais próximo, de forma a permitir trocas estratégicas de informações. Como conseqüência dessa confluência, os processos de negócios da CS requerem uma sintonia com esses mecanismos, para que possa ser implementada a GCS da organização.

O comportamento de Beta e Gama quanto às ações ou mudanças em suas gestões internas exemplificam os impactos da EP de Alfa sob seus processos de negócios. Beta realizou algumas adaptações em seu sistema produtivo para atender os objetivos de Alfa, os quais se destacam: (a) formação de uma equipe de engenharia voltada para pesquisas de desenvolvimento de componentes específicos aos produtos de Alfa - em função da prioridade de inovação de Alfa, facilitando o processo DP, (b) planejamento da produção programada para entregas via kanban - em função da prioridade custos de Alfa, apoiando os processos GFP e GD e (c) implementação de um software para facilitar a interface de programação entre Beta e Alfa - facilita à implementação da prioridade serviço e a integração entre os processos de GRF e GD. Com essas mudanças comportamentais Beta contribui na execução dos processos de negócios de Alfa. Gama é uma empresa com um perfil proativo na área de inovação em produtos e para atender Alfa não precisou de mudanças consideráveis em seu sistema produtivo. As práticas de Gama que facilitam a sua integração com Alfa são: (a) possuir engenheiros de aplicação trabalhando em equipe com a engenharia do cliente para desenvolver novos protótipos - em função da prioridade inovação de Alfa, facilitando o processo de DP, (b) verticalizar ao máximo os processos para diminuir o time to market - auxilia na obtenção das prioridades de custo e inovação e na execução dos processos de GFP e DP e (c) 
planejamento da produção programada para entregas via kanban - em função da prioridade custos de Alfa, apoiando os processos de GFP e GD.

Pelo exposto, percebe-se que a EP de Alfa, a partir do conjunto de mecanismos, facilita a execução dos processos de negócios entre Alfa e Beta e Alfa e Gama. Uma vez que Alfa foca em custos, flexibilidade em inovação e serviço, esses orientam os critérios de seleção de fornecedores; a forma de se relacionar com eles; o tipo de informações trocar com cada um; e com quais deles desenvolver conjuntamente produtos. Esses mecanismos modelam a forma de executar os processos de negócios e, por conseguinte, a forma como os fornecedores cooperam na implementação da GCS de Alfa.

Pode-se concluir que as orientações das atuações de Beta e Gama para executar os processos de negócios comuns a Alfa têm relação com a EP daquela. Seja a partir de adaptações, ou atuando a sua maneira, Beta e Gama respondem às demandas de Alfa por inovação de produto (equipe de engenheiros dedicados e verticalização da produção), redução de custos (entregas via kanban e verticalização da produção) e apoio aos fornecedores (desenvolvimento conjunto de produtos e implementação de software de TI). A figura 3 ilustra esses resultados.

Figura 3: Relações entre EP de Alfa e os processos de negócios estabelecidos com Beta e Gama.

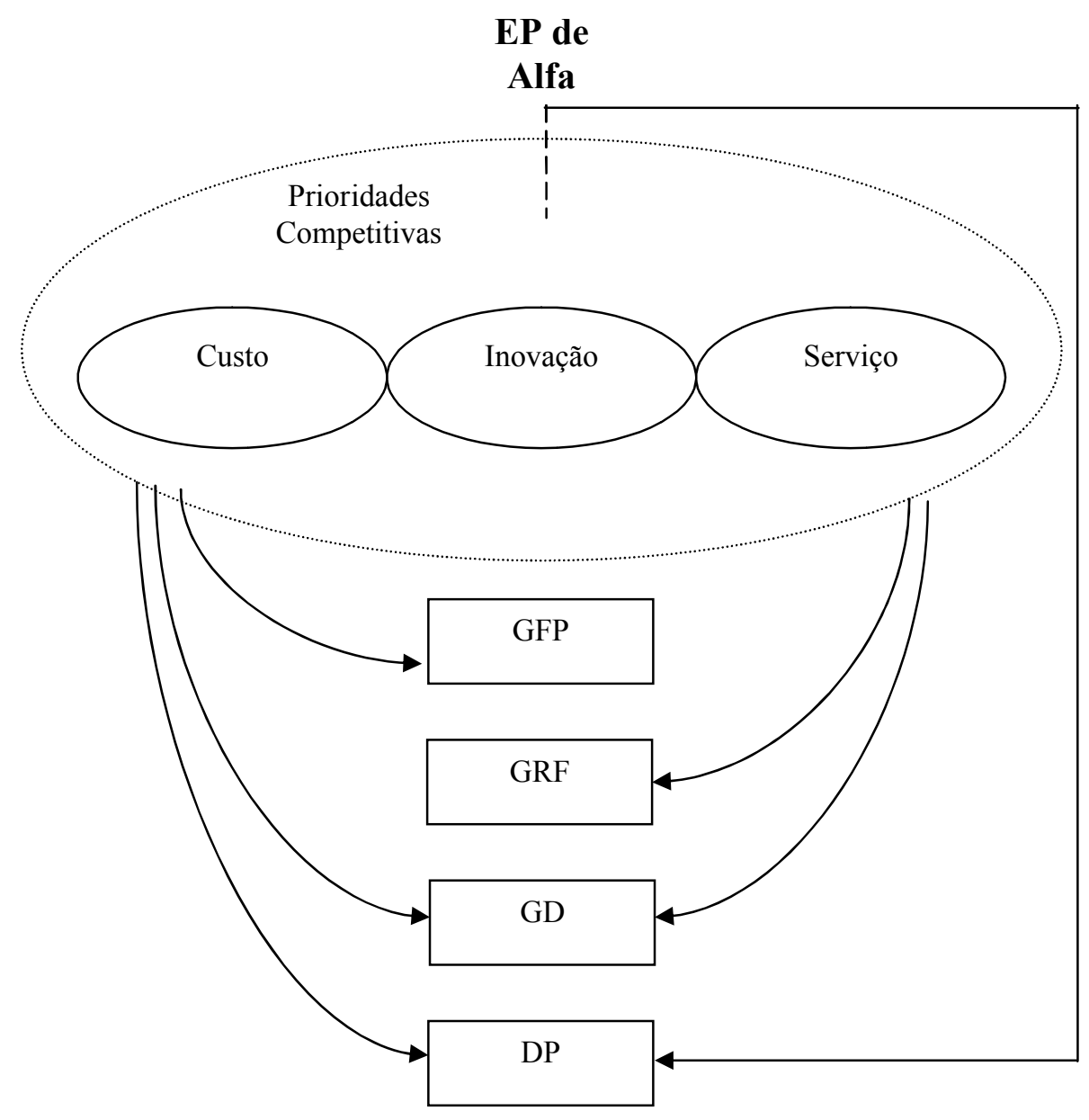

Conjunto de mecanismos

Fonte: Elaboração própria (2007). 


\section{Considerações Finais}

Este trabalho buscou identificar uma forma de entender como a EP de uma empresa afeta a sua GCS mantida com fornecedores de primeira camada. A partir de um estudo de caso com três empresas que representam dois elos a montante de uma cadeia, evidenciou-se que as prioridades competitivas da empresa focal orientam o perfil do conjunto de mecanismos, conjunto de atividades de áreas funcionais (inter-funcionais) de uma organização, e esses influenciam a forma como os fornecedores gerenciam seus processos de negócios. Uma vez que os processos de negócios entre Alfa, Beta e Gama se complementam, a GCS da empresa focal se torna possível. Os resultados alcançados por esta pesquisa se justificam pelos argumentos de Mentzer et al. pois há a constatação de que houve mudança da gestão organizacional interna das empresas fornecedoras (entregas programadas por cartão kanban e equipe de engenheiros dedicados aos projetos de produtos da montadora) no intuito de estabelecer cooperação pela eficiência conjunta e integração de processos de negócios, apontando que os fornecedores de primeira camada buscaram se adequar a GCS estabelecida por Alfa. Além do mais, seguindo a proposta de Lambert e Cooper (2000), esta pesquisa identificou e delimitou membros de uma CS, em função da importância da montadora em seu setor e em função de certas especificidades dos componentes fornecidos pelos seus fornecedores, relacionou os processos de negócios executados conjuntamente e como os fornecedores suportaram com suas ações a execução de tais processos, pode-se afirmar então que foi possível verificar a implementação da GCS da montadora Alfa.

As principais contribuições deste trabalho seguem em três direções: (a) a delimitação EP da empresa focal, a partir das prioridades competitivas, pode ser considerada como uma diretriz na definição do perfil de atividades inter-funcionais no nível da empresa tendo reflexo nas atividades externas, os processos de negócios, de forma a influenciar na GCS da empresa focal, (b) o fato dos processos de negócios entre os atores da cadeia se integrarem tendo como resultado a cooperação dos fornecedores com a empresa focal demonstra que conjunto de mecanismos é uma forma de orientar a GCS a apoiar a EP da empresa focal e (c) o conjunto de mecanismos demonstrou ser um artifício capaz de relacionar o perfil da EP da empresa com os processos de negócios e conseqüentemente relacionar a EP com a GCS. Esta última responde a proposta de pesquisas futuras de Cerra et al.,(2007) de indicar como elementos operacionais se combinam para apoiar as estratégias competitivas e de GCS das empresas. 
Uma das limitações deste trabalho tange o método de pesquisa, estudo de caso, o qual não proporciona maiores generalizações dos resultados. No entanto, deve-se considerar que apesar de ser apenas um caso, este é representativo em seu setor (eletrodomésticos) no Brasil, pois foram estudados e analisados a montadora líder e dois de seus fornecedores críticos em termos de desempenho de produtos e especificidade do componente.

Como sugestão de trabalhos futuros derivados deste propõe-se que se faça um estudo quantitativo para confirmar a validade do conjunto de mecanismos como um artifício para entender o relacionamento entre EP e GCS a partir da execução de processos de negócios conjuntos e também, que se desenvolvam outros estudos de casos utilizando-se a estrutura dos pressupostos metodológicos adotados neste trabalho, só que em empresas de outros setores para que se possa fazer análises comparativas, pois segundo Fisher (1997) cada tipo de produto requer uma estrutura de CS que suporte as operações necessárias para atender ao perfil do cliente daquele produto.

\begin{abstract}
The objective of this study is to understand how an assembly company that is considered a focal company in the chain of Brazilian white goods sector can influence the supply chain management established with its first tier suppliers. This is an exploratory qualitative study in which the information was gathered through direct observations, documents' retention, and data from interviews held with management-level employees of the sales and product development areas of the focal company and of the production area of the suppliers' companies. This study indicates that the operations strategy, OS, of the focal company influences the supply chain management and that the common business processes shared by its suppliers are a way to verify the truth of such statement. Moreover, the suppliers cooperate closely with the focal company when complementing their business processes and consequently supporting the company to pursue its operations strategy. A set of mechanisms to aid the comprehension of how the operations strategy can affect the business processes and therefore to achieve the result of this research were adopted.
\end{abstract}

Keywords - operations strategy; supply chain management; business processes; white goods.

\title{
Referência
}

BARRATT, M. Understanding the meaning of collaboration in the supply chain. Supply Chain Management: An International Journal, v. 9, n. 1, p. 30-42, 2004.

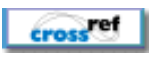

BIROU, L. M.; FAWCETT, S. E. Supplier involvement in integrated product development: a comparison of US and european practices. Intenational Journal of Physical Distribution \& Logistics Management, v. 24, n. 5, p. 4-14, 1994. cross ${ }^{\text {ref }}$

CERRA, A. L.; MAIA, J. L.; ALVES FILHO, A. G. Aspectos estratégicos, estruturais e relacionais de três cadeias de suprimentos automotivas. Revista Gestão \& Produção, v. 14, n. 2, p. 253-265, 2007.

CHISTOPHER, M. Logistics and supply chain management. Londres: Prentice Hall, 1998. 
CHOPRA. S.; MEINDL. P. Gerenciamento da cadeia de suprimentos - estratégias, planejamento e operações. São Paulo: Prentice Hall, 2003.

CLARO, D. P.; CLARO, P. B. O.; HAGELAAR, G. Coordinating collaborative join efforts with suppliers: the effects of trust, transaction specific investment and information network in the dutch flower industry. Supply Chain Management: An International Journal, v. 11, n. 3, p. 216-224, 2006.

crossef

COOPER, M. C.; LAMBERT, D. M.; PAGH, J. D. Supply chain management: more than a new name for logistics. International Journal of Logistics Management, v. 8, n. 1, p. 1-14, 1997.

crossef

CROXTON, K. L.; GARCIA-DASTUGUE, S. J.; LAMBERT, D. M. The supply chain management processes. International Journal of Logistics Management, v. 12, n. 2, p. 13-36, 2001.

cross ${ }^{\text {ref }}$

DEMETER, K.; GELEI, A.; JENEI, I. The effect of strategy on supply chain configuration and management practices on the basis of two supply chains in the Hungarian automotive industry. International Journal of Production Economics, v. 104, p. 555-570, 2006.

cross ${ }^{\text {ref }}$

FINE, C. H.; HAX, A. C. Manufacturing strategy: a methodology and an illustration. Interfaces, v. 15, n. 6, p. 2846, 1985.

cross ${ }^{\text {ref }}$

FISHER, M. L. What is the right supply chain for your product? Harvard Business Review, March-April, p. 105$116,1997$.

FURTADO, G. A. P. Critérios de seleção de fornecedores para relacionamentos de parcerias: um estudo em empresas de grande porte. 2005. Dissertação (Mestrado em Administração) - Departamento de Administração Faculdade de Economia, Administração e Contabilidade da Universidade de São Paulo, São Paulo, 2005.

GARVIN, D. A. Manufacturing strategic planning. California Management Review Summer, p. 85-106, 1993.

GATTORNA, J. L. Strategic supply chain alignment. Aldershot: Gower, 1998.

GHODESWAR, B.; VAIDYANATHAN, J. Business process outsourcing: an approach to gain access to world-class capabilities. Business Process Management Journal, v. 14, n. 1, p. 23-38, 2008.

cross ${ }^{\text {ref }}$

HARWICK, T. Optimal decision-making for the supply chain. APICS - The Performance Advantage, v. 7, n.1, p. $42-44,1997$.

HAYES, R. H.; WHEELWRIGHT, S. C.; CLARK, K. Dynamic manufacturing. New York: Free Press, 1988

HAYES, R.H.; WHEELWRIGHT, S.C. Restoring our competitive edge - competing through manufacturing. USA: John Wiley \& Sons, 1984.

LAMBERT, D. M.;COOPER, M. C. Issues in supply chain management. Industrial Marketing Management, v. 29, p. $65-83,2000$

crossef

LEE-MORTIMER. A. L. Supplier integration. World Class Design to Manufacture, v. 1, n. 6, p. 39-43, 1994.

cross ${ }^{\text {ref }}$

LEONG, G.K.; SNYDER, D.L.; WARD, P.T. Research in the process and content of manufacturing strategy. Omega International Journal of Management Science, v. 18, n. 2, p. 109-122, 1990.

cross'

LI, J.; SIKORA, R.; SHAW, M. J.; TAN, G. W. A strategic analysis of inter organizational information sharing. Decision Support Systems, v. 42, p. 251-266, 2006.

cross ref 
MAIA, J. L.; CERRA, A. L.; ALVES FILHO, A. G. Inter-relações entre estratégia de operações e gestão da cadeia de suprimentos: estudos de caso no segmento de motores para automóveis. Revista Gestão e Produção, v. 12, n. 3, p. 377-391, 2005.

MENTZER, J. T.; DEWITT, W.; KEEBLER, J.; MIN, S.; NIX, N.; SMIITH, C.; ZACHARIA, Z. Defining supply chain management. Journal of Business Logistics, v. 22, n. 2, p. 1-25 2001.

PRAHALAD, C. K.; HAMEL, G. The core competence of the corporation. Harvard Business Review, v. 68, n. 3, p. 79-91, 1990.

QUINN, J. B. Strategies for change. In: QUINN, J. B.; MINTZBERG, H.; JAMES, R. M. The strategy process: concepts, contexts, and cases. Englewood Cliffs : Prentice-Hall, 1988. p. 2-9

SAHAY, B. S.; GUPTA, F. N. D.; MOHAN, R. Managing supply chains for competitiveness: the indian scenario. Supply Chain Management: An International Journal, v. 11, n. 1, p. 15-24, 2006.

cross ${ }^{\text {ref }}$

SEURING, S. A. Strategic supply chain management - from focused factories to focused supply chains. In: Seuring, Stefan. A.; Muller, Martin.; Goldbach, Maria.; Schneidewind, Uwe (eds.): Strategy and Organization in Supply Chains. Heidelberg: Physica, 2003, p. 181-196.

SKINNER, W. Manufacturing-missing link in corporate strategy. Harvard Business Review May/Jun, p. 136-145, 1969.

SLACK, N.; CHAMBERS, S.; JOHNSTON, R. Administração da produção. São Paulo: Atlas, 2002.

VACHON, S.; HALLEY, A.; BEAULIEU, M. Aligning competitive priorities in the supply chain: the role of interactions with suppliers. International Journal of Operations \& Production Management, v. 29, n. 4, p. 322-344, 2009.

cross ${ }^{\text {ref }}$

VAN ECHTELT, F. E. A.; WYNSTRA, J. Y. F.; VAN WEELE, A. J.; DUYSTERS. Managing supplier involvement in new product development: a multiple-case study. Social Science Research Network, artigo submetido em 2006 para publicação, aguarda revisão.

VOSS, C. A. Alternative paradigms for manufacturing strategy. International Journal of Operations \& Production Management, v. 15, n. 4, p. 5-16, 1995.

cross ${ }^{\text {ref }}$

VOSS, C.; TSIKRIKTSIS, N.; FROHLICH, M. Case research in operational management. International Journal of Operations \& Production Management. v. 22, n. 2, p.195-219, 2002.

cross ${ }^{\text {ref }}$

YIN, R. Estudo de casos: planejamento e métodos. São Paulo: Bookman, 2004.

\section{DADOS DOS AUTORES}

Nome completo: Ana Beatriz Lopes de Sousa Jabbour

Filiação institucional: Universidade Federal de São Carlos - Campus Sorocaba

Departamento: Engenharia de Produção

Função ou cargo ocupado: Professora Assistente

Endereço completo para correspondência (bairro, cidade, estado, país e CEP): Rodovia João Leme dos Santos, Km 110 - SP-264. Bairro do Itinga. Sorocaba - São Paulo - Brasil. CEP 18052-780

Telefones para contato: (15) 3229-5962 
e-mail:beatriz@dep.ufscar.br

Nome completo: Alceu Gomes Alves Filho

Filiação institucional: Universidade Federal de São Carlos

Departamento: Engenharia de Produção

Função ou cargo ocupado: Professor Titular

Endereço completo para correspondência (bairro, cidade, estado, país e CEP): Rodovia Washington Luís, km 235 - SP-310. São Carlos - São Paulo - Brasil. CEP 13565-905

Telefones para contato: (16) 3351-9523

e-mail:alceu@power.ufscar.br

Nome completo: Maria Rita Pontes Assumpção

Filiação institucional: Universidade Católica de Santos

Departamento: Gestão de Negócios

Função ou cargo ocupado: Professora do mestrado

Endereço completo para correspondência (bairro, cidade, estado, país e CEP): Rua Carvalho de Mendonça número, 144, $5^{\circ}$ andar. Vila Matias. 11070-906 - Santos, SP - Brasil

Telefones para contato: (13) 3205-5555

e-mail: rita.assumpcao.@unisantos.br

Recebido para publicação em: 08/10/2009

Aceito para publicação em: 04/12/2009 